\title{
Matrix Converter Using Direct Svm Method with Input Power Factor Compensation
}

\author{
${ }^{1}$ M. K. Kiran (M. Tech), ${ }^{2}$ A.Srinivasulu. M. E. \\ ${ }^{I}$ Student, ${ }^{2}$ Assistant Professor,Electrical and Electronics Engineering Madanapalli Institute of Technology and \\ Science
}

\begin{abstract}
The matrix converter (MC) for motor drive applications and energy conversion systems is steadily increasing due to its main advantage of performing a direct coupling between two three-phase alternating current sources without the need of an intermediate direct current bus. The input filters characteristics design for matrix converter system: to reduce the high harmonic components at the main power supply current and to improve the input voltage distortion for matrix converter, the unity power factor at the main power supply side is only achieved at the high output load and decreases greatly at the light load conditions. This paper proposes a new direct space vector modulation (DSVM) method to achieve the required displacement angle between input voltage and input current of matrix converter. A new switching strategy is introduced based on the maximum compensated angle and for the controllable input power factor of matrix converter. The power factor compensation algorithms using the new DSVM method to achieve the maximum IPF are presented, in which compensation algorithm I is based on using the input filter and power supply parameters to estimate the optimal compensated angle. Compensation algorithm II is subsequently proposed using a proportional-integral controller to overcome drawbacks presented in compensation algorithm I. Simulation results with inductive load $(R L)$ are shown to validate the effectiveness of the proposed method.
\end{abstract}

Keywords - Direct space vector modulation (DSVM) method, input filter, matrixconverter (MC).

\section{Introduction}

In past two decades, the progress of power device technology and the development of large power integrated circuits have reviewed direct AC-AC power conversion technologies. This converter fulfills all the requirements of the conventionally used rectifier/ dc link/ inverter structures and provides an efficient way to convert electric power for motor drives, UPS, VF generators and reactive energy control. In general, the desirable characteristics of AC to AC converters are

1) Sinusoidal input and output wave forms with minimal higher order harmonics and no sub-harmonics,

2) Bidirectional energy flow capability,

3) Minimal energy storage requirements (minimal size reactive component),

4) A controllable power factor,

5) Operations in all four quadrants of the torque-speed plane due to the regenerative capability,

6) High reliability and long life due to the absence of bulky electrolytic capacitors,

7) Smaller and lighter design than other regeneration inverter with equivalent power ratings.

The input filter designs should guarantee the near unity power factor operation at the power source side, to improve the main input current quality which has the sinusoidal waveforms with containing low high harmonic components and to reduce the input voltage distortion which supplies to matrix converter module. The presence of input filter in the direct ac-ac power conversion, which has no energy storage, can cause the instability during operations. The input filter design for sliding mode controlled MC considered the maximum allowable displacement angle introduced by the filter and the controllable IPF capability, as well as the ripple presented in capacitor voltages.

The input filter still exists, which results in a displacement angle between input line-to-neutral voltage and input line current at the main power supply. Consequently, the IPF at the power supply could be far from the desired power factor of unity. In particular, in the case of low-output-power condition, the IPF at the power supply would decrease significantly. In order to overcome this problem, we proposed a new direct space vector modulation (DSVM) method based on the maximum controllable displacement angle between the input current and input voltage of the MC. The new DSVM method was developed by using a new pulse width modulation (PWM) switching pattern. In this paper, two IPF compensation algorithms using the new DSVM are proposed to improve the IPF of the MC. First, a compensation algorithm based on the calculation of the optimal compensated angle is analyzed and discussed. This compensation algorithm provides a fast response which allows high IPF achievement. However, the accuracy of this algorithm depends on some of the MC's parameters, including power supply and $L C$ filter values. Subsequently, another compensation algorithm is suggested to overcome the drawbacks presented in the first compensation algorithm. This compensation 
algorithm is based on a proportional-integral (PI) controller usage for power factor control. Aside from the flexible adjustment capability of the power factor that this compensation algorithm can provide, its performance is independent on the MC's configuration.

System responses in both transient and steady-state operations are presented in this paper. Comparison between the two IPF compensation algorithms is also analyzed and summarized in details. Simulation results are presented illustrating better performances of the proposed compensation algorithms using the new DSVM method.

\section{Input filter analysis of MC}

The input filter is designed to filter high harmonic components of the input current and to reduce the input voltage distortion supplied to the MC.The equivalent circuit of a single-phase input filter for matrix converter as shown in Fig.1.

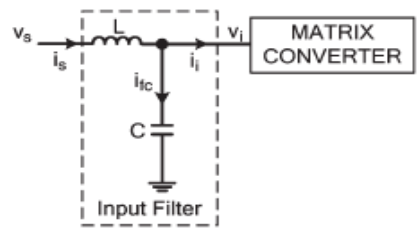

Fig.1.MC equivalent circuit of single phase input filter.

The following are basic equations regarding input and output voltages, and currents of the MC:

$$
\begin{aligned}
& \boldsymbol{v a} \mathbf{a}=V \mathfrak{m} \cos (\omega t), v c=V \mathfrak{m} \cos \left(\omega t-\frac{4 \pi}{3}\right), v \mathfrak{b}=V \mathfrak{m} \cos \left(\omega t-\frac{2 \pi}{3}\right) \\
& \boldsymbol{v} i=2\left(\boldsymbol{v} \mathfrak{a}+\boldsymbol{v} \mathfrak{b} e^{j 2 \pi / 3}+\boldsymbol{v} \boldsymbol{c} e^{j 4 \pi / 3}\right) / 3=V i e^{j \propto i} \\
& \boldsymbol{v}=2\left(\boldsymbol{v} \mathrm{A}+\boldsymbol{v} \mathrm{B} e^{j 2 \pi / 3}+\boldsymbol{v} \mathrm{C} e^{j 4 \pi / 3}\right) / 3=\mathrm{V}^{j \alpha_{\alpha}} \\
& i \mathrm{i}=2\left(i \mathbf{a}+i \mathbf{b} e^{j 2 \pi / 3}+i \boldsymbol{c} e^{j 4 \pi / 3}\right) / 3=\mathrm{I} i e^{j \beta i} \\
& i=2\left(i \mathrm{~A}+i \mathrm{~B} e^{j 2 \pi / 3}+i \mathrm{C} e^{j 4 \pi / 3}\right) / 3=\mathrm{I} i e^{j \beta}
\end{aligned}
$$

From Fig. 1, the following equations are obtained:

$$
\begin{aligned}
& v \mathfrak{a}=v_{\mathfrak{s}}-L(d i \mathfrak{s} / d t) \\
& \text { if } \boldsymbol{c}=\mathrm{C}(\mathrm{d} \boldsymbol{v} i / \mathrm{dt})
\end{aligned}
$$

$$
i \mathfrak{s}=i f c+i i
$$

Considering the power factor on the power supply side, the power supply frequency in (1) will be taken as the fundamental frequency. The MC control algorithm guarantees the load control requirements and unity power factor at the power supply side as well. Then, (6) can be rewritten as follows:

$$
\begin{aligned}
\mathrm{V} \mathfrak{s} & =V \mathfrak{s} e^{j 0} \\
\mathrm{~V} i & =\mathrm{V} \mathfrak{s}-\mathrm{j} \omega L I \mathfrak{s} \\
& =\sqrt{V \mathfrak{s}^{2}+\omega L I \mathfrak{s}^{2}} \boldsymbol{e}^{-j \tan ^{-1}\left(\frac{\omega L I \mathfrak{s}}{V \mathfrak{s}}\right)} \\
\mathrm{I} i & =\left(1-\omega^{2} \mathrm{LC}\right) \mathrm{I} \mathfrak{s}-\mathrm{j} \omega C V \mathfrak{s} \\
& =\sqrt{\left[\left(1-\omega^{2} \mathrm{LC}\right) \mathrm{I}\right]^{2}+(\omega C V \mathfrak{s})^{2}} e^{-j \tan ^{-1}\left[\frac{\omega C V \mathfrak{s}}{\left(1-\omega^{2} \mathrm{LC}\right) \mathrm{Is}}\right]}
\end{aligned}
$$

Finally, the input current of the power supply always leads the input current of the MC with the following displacement angle:

$$
\delta=\varphi i \mathfrak{s} / i \mathrm{i}=\tan ^{-1}\left[\frac{\omega C V \mathfrak{s}}{\left(1-\omega^{2} \mathrm{LC}\right) \mathrm{Is}}\right]
$$

From (8), the displacement angle depends on three parameters: the $L$ and $C$ values of the input filter and the fundamental input current amplitude $I s$, which depends on the output load conditions of the MC. In order to minimize this displacement angle, small $L C$ values are used in the practical MC system. However, with small $L C$ values, the main power supply current contains higher harmonic components resulting from the well known higher cutoff frequency $1 /(2 \pi \sqrt{L C})$

To achieve a maximum IPF at the main power supply, the SVM method, which guarantees the input current and input voltage of the MC to be in phase, must be revised to compensate for the displacement angle caused by the input filter. 
3.1 Space Vectors of MC

\section{New DSVM Method}

The three-phase MC module includes nine bidirectional switches, as shown in Fig. 2.

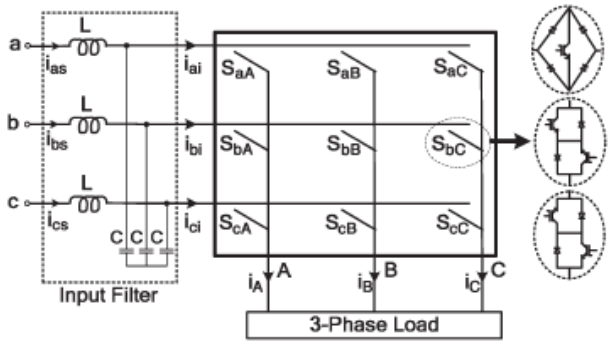

Fig.2. Structure of a three-phase ac-ac MC.

There are 27 switching configuration states (SCs), however, only 21 SCs can be used to implement the modern control algorithms for matrix converter such as Space Vector Modulation, DTC method etc... As shown in table I: group I $( \pm 1, \pm 2 \ldots \pm 9)$ consists of the SCs which have two output phases connected to the same one of the other input phase, group II $(0 \mathrm{a}, 0 \mathrm{~b}, 0 \mathrm{c})$ consists of the SCs which have all output phases connected to a common input phase. For each SC, the corresponding line-to-neutral voltage vector and input line current vector have fixed directions as represented in Fig. 3. The other 6 SCs have the output phases connected to the different input phases. In this case, the output voltage vector and input current vector have variable directions and can not be usefully used.

TABLE I

\begin{tabular}{|c|c|c|c|c|c|c|c|}
\hline Group & Vector & A & $\mathrm{B}$ & $V_{o}$ & $\alpha_{v}$ & $I_{i}$ & $\beta_{i}$ \\
\hline \multirow{18}{*}{ I } & +1 & $a \quad b$ & $b$ & $2 / 3 \mathrm{v}_{\mathrm{ab}}$ & 0 & $2 / \sqrt{ } 3 i_{A}$ & $-\pi / 6$ \\
\hline & -1 & b : & $\mathrm{a}$ : & $-2 / 3 \mathrm{v}_{\mathrm{ab}}$ & 0 & $-2 / \sqrt{ } 3 i_{A}$ & $-\pi / 6$ \\
\hline & +2 & b c & c c & $2 / 3 \mathrm{v}_{\mathrm{bc}}$ & 0 & $2 / \sqrt{ } 3 i_{A}$ & $\pi / 2$ \\
\hline & -2 & c b & $b \mathrm{~b}$ & $-2 / 3 \mathrm{v}_{\mathrm{bc}}$ & 0 & $-2 / \sqrt{ } 3 i_{A}$ & $\pi / 2$ \\
\hline & +3 & c a & $\mathrm{a} a$ & $2 / 3 \mathrm{v}_{\mathrm{ca}}$ & 0 & $2 / \sqrt{3} i_{\Lambda}$ & $7 \pi / 6$ \\
\hline & -3 & a c & $\mathrm{cc}$ & $-2 / 3 \mathrm{v}_{\mathrm{ca}}$ & 0 & $-2 / \sqrt{ } 3 i_{A}$ & $7 \pi / 6$ \\
\hline & +4 & b a & $\mathrm{ab}$ & $2 / 3 \mathrm{v}_{\mathrm{ab}}$ & $2 \pi / 3$ & $2 / \sqrt{ } 3 i_{B}$ & $-\pi / 6$ \\
\hline & -4 & $\mathrm{a} \mathrm{b}$ & $\mathrm{ba}$ & $-2 / 3 v_{a b}$ & $2 \pi / 3$ & $-2 / \sqrt{ } 3 i_{B}$ & $-\pi / 6$ \\
\hline & +5 & $\mathrm{c} \mathrm{b}$ & $\mathrm{bc}$ & $2 / 3 v_{b c}$ & $2 \pi / 3$ & $2 / \sqrt{ } 3 i_{B}$ & $\pi / 2$ \\
\hline & -5 & $\mathrm{~b} \mathrm{c}$ & $\mathrm{c} \mathrm{b}$ & $-2 / 3 \mathrm{v}_{\mathrm{bc}}$ & $2 \pi / 3$ & $-2 / \sqrt{3} i_{B}$ & $\pi / 2$ \\
\hline & +6 & a c & c a & $2 / 3 \mathrm{v}_{\mathrm{ca}}$ & $2 \pi / 3$ & $2 / \sqrt{ } 3 i_{B}$ & $7 \pi / 6$ \\
\hline & -6 & $\mathrm{c} a$ & $\mathrm{a} c$ & $-2 / 3 \mathrm{v}_{\mathrm{ca}}$ & $2 \pi / 3$ & $-2 / \sqrt{ } 3 i_{B}$ & $7 \pi / 6$ \\
\hline & +7 & $\mathrm{~b} \mathrm{~b}$ & $\mathrm{~b} a$ & $2 / 3 \mathrm{v}_{\mathrm{ab}}$ & $4 \pi / 3$ & $2 / \sqrt{ } 3 i_{C}$ & $-\pi / 6$ \\
\hline & -7 & $\mathrm{a} a$ & $\mathrm{ab}$ & $-2 / 3 v_{a t h}$ & $4 \pi / 3$ & $-2 / \sqrt{3} i_{C}$ & $-\pi / 6$ \\
\hline & +8 & c c & $\mathrm{c} \mathrm{b}$ & $2 / 3 \mathrm{v}_{\mathrm{bc}}$ & $4 \pi / 3$ & $2 / \sqrt{3} \mathbf{i}_{\mathrm{c}}$ & $\pi / 2$ \\
\hline & -8 & b b & $b$ c & $-2 / 3 v_{b c}$ & $4 \pi / 3$ & $-2 / \sqrt{ } 3 i_{c}$ & $\pi / 2$ \\
\hline & +9 & a a & $\mathrm{a}$ & $2 / 3 \mathrm{v}_{\mathrm{ca}}$ & $4 \pi / 3$ & $2 / \sqrt{3} i_{C}$ & $7 \pi / 6$ \\
\hline & -9 & c c & $\mathrm{c} a$ & $-2 / 3 \mathrm{v}_{\mathrm{ca}}$ & $4 \pi / 3$ & $2 / \sqrt{3} \mathrm{i}_{\mathrm{C}}$ & $7 \pi / 6$ \\
\hline \multirow{3}{*}{ II } & $0_{a}$ & a a & $\mathrm{a} a$ & 0 & - & 0 & - \\
\hline & $0_{\mathrm{b}}$ & $\mathrm{b} \mathrm{b}$ & $\mathrm{b} \mathrm{b}$ & 0 & - & 0 & - \\
\hline & $0_{\varepsilon}$ & c c & $c c$ & 0 & - & 0 & - \\
\hline
\end{tabular}

3.2 New Direct SVM Method for Matrix Converter

For the sake of explaining the new DSVM method, we assume both the desired output voltage and the input voltage space vectors to be located in sector 1 without missing the generality of the analysis $(\mathrm{kv}=1, \mathrm{ki}=1)$. Where $\mathrm{kv}$ and ki are the output voltage vector sector and the input current vector sector, respectively.

Case 1: The displacement angle makes the desired input current vector and input voltage vector locate on the same sector, in Fig. 3(b).

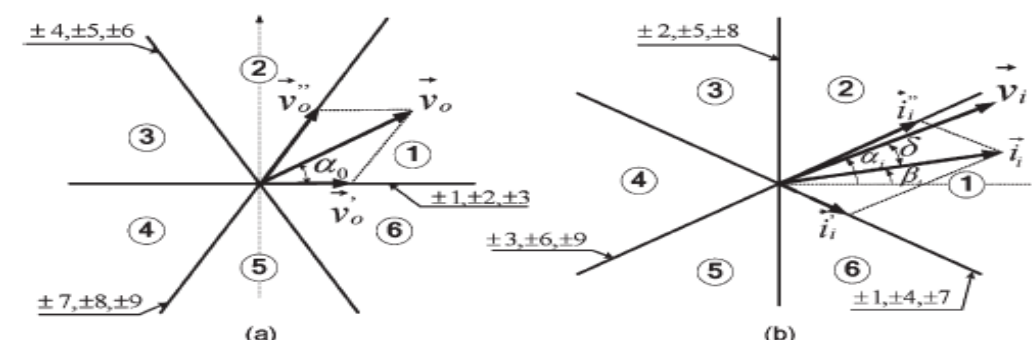

(a)

(b)

Fig. 3. (a) Output line-to-neutral voltage vector. (b) Input line current vector. 
The desired output voltage vector $\underset{v}{\rightarrow}$ is generated from two vectors $\underset{v}{\vec{\prime}}$ and $\underset{v}{\vec{v}}$ ". To match the vector direction as $\rightarrow$ "among the six possible SCs $( \pm 7, \pm 8, \pm 9)$ that have the output voltage vector in the same direction of $\underset{v}{\vec{v}}$ “, only two higher voltage magnitude vectors are considered to generate which must maintain the input current vector direction $i i$ to be inside sector 1 and lag behind the input voltage vector with a certain angle $\delta=\alpha i-\beta i$. In order to approach the given conditions, SCs -7 and +9 are selected to drive the MC, and from Fig. 3, the duty ratios of SCs $-7(d 1)$ and $+9(d 2)$ should satisfy the following relationship:

$\mathrm{d}_{1} /(\sin \Pi / 6-\beta \mathbf{i})=\mathrm{d}_{2} / \sin (\Pi / 6+\beta i)$

$\mathrm{d}_{1}(-2 v \mathrm{ab} / 3)+\mathrm{d}_{2}(2 v \mathrm{ca} / 3)=v_{0}$ "

Solving (9) under the given condition $\delta=\alpha i-\beta i$, the duty ratios for SCs -7 and +9 are respectively as follows:

$d_{1}=2 q \sin [\alpha o-(k v-1) \Pi / 3] \sin \left[\frac{\Pi}{6}-(\alpha i-\delta-(k i-1) \Pi / 3)\right] / \sqrt{3} \cos (\delta)$

$d_{2}=2 q \sin [\alpha o-(k v-1) \Pi / 3] \sin \left[\frac{\Pi}{6}+(\alpha i-\delta-(k i-1) \Pi / 3)\right] / \sqrt{3} \cos (\delta)$

By similar analysis, the SCs selected to obtain $\_v_{-} o$ are $+1(d 3)$ and $-3(d 4)$ with the following duty ratios:

$d_{3}=2 q \sin \left[\left(\frac{k v \Pi}{3}-\alpha o\right] \sin \left[\frac{\Pi}{6}-(\alpha i-\delta-(k i-1) \Pi / 3)\right] / \sqrt{3} \cos (\delta)\right.$

$d_{4}=2 q \sin \left[\left(\frac{k v \Pi}{3}-\alpha o\right] \sin \left[\frac{\Pi}{6}+(\alpha i-\delta-(k i-1) \Pi / 3)\right] / \sqrt{3} \cos (\delta)\right.$

Finally, zero SC $(d 5)$ is applied to complete the sampling period

$\mathrm{d}_{5}=1-\left(d_{1}+d_{2}+d_{3}+d_{4}\right)$

$\mathrm{d}_{5}=2 \mathrm{q} \cos [\alpha o-\{(2 k v-1) \Pi / 6\}] \cos [\alpha i-\delta-(k i-1) \Pi / 3] / \sqrt{3} \cos (\delta)$

Where $d_{1}, d_{2}, d_{3}, d_{4}$, and d5 are the duty ratios of four active SCs (in this case, $-7,+9,+1$, and -3 ) and zero SC, Respectively, and $\mathrm{q}=\mathrm{Vo} / \mathrm{Vm}$. is the voltage transfer ratio.

Table II shows all switching patterns that can be used in the DSVM method if the desired input current vector is located in the same sector with the input voltage vector.

TABLE II

SWITCHING PATTERNS FOR THE INPUT VOLTAGE AND CURRENT VECTORS LOCATED IN THE SAME SECTOR

\begin{tabular}{|c|c|c|c|c|c|c|c|}
\hline & \multicolumn{6}{|c|}{ Output voltage vetor sector } \\
\hline & & 1 & 2 & 3 & 4 & 5 & 6 \\
\hline \multirow{6}{*}{ 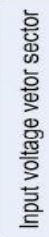 } & 1 & $+9+$ & $+9-8-3+2$ & $-8+7+2-1$ & $+7-9-1+3$ & $-9+8+3-2$ & $+8-7-2+1$ \\
\hline & 2 & $-4-6=7+9$ & $-6+5+9-8$ & $+5-4-8+7$ & $-4+6+$ & $+6-5-9+8$ & $-5+4+8-7$ \\
\hline & 3 & +3 & $+3-2-6+5$ & $-2+1+5-4$ & $+1-3-4+6$ & $-3+2+6-5$ & $+2-1-5+4$ \\
\hline & 4 & $7-9-1+3$ & $-9+8+3-2$ & $+8-7-2+1$ & $-7+9$ & $+9-8-3$ & $-8+$ \\
\hline & 5 & $+6+$ & $+6-5-9$ & $-5+4+8-7$ & $+4-6-7+9$ & $-6+5+9-8$ & $+5-4-8+$ \\
\hline & 6 & $+1-3-4+6$ & $-3+2+6$ & $+2-1-5+4$ & $-1+3+$ & $+3-2-6+5$ & $-2+1+$ \\
\hline \multicolumn{2}{|c|}{ Duty ratios } & $d_{1} d_{2} d_{3} d_{4}$ & $d_{1} d_{2} d_{3} d_{4}$ & $d_{1} d_{2} d_{3} d_{4}$ & $d_{1} d_{2} d_{3} d_{4}$ & $d_{1} d_{2} d_{3} d_{4}$ & $d_{1} d_{2} d_{3} d_{4}$ \\
\hline
\end{tabular}

Case 2: The displacement angle makes the desired input current vector and input voltage vector locate on the different sectors. Without missing the generality, assume the input voltage vector is located in sector 1 and desired input current vector is located in sector 6 with the different phase $\delta$, in Fig. 4.

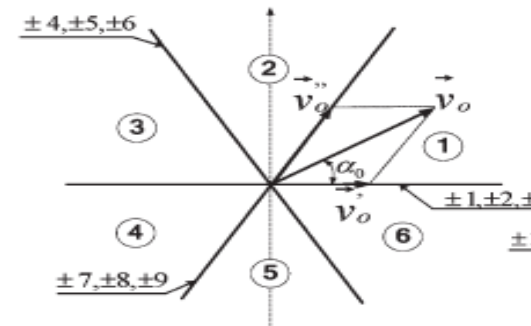

(a)

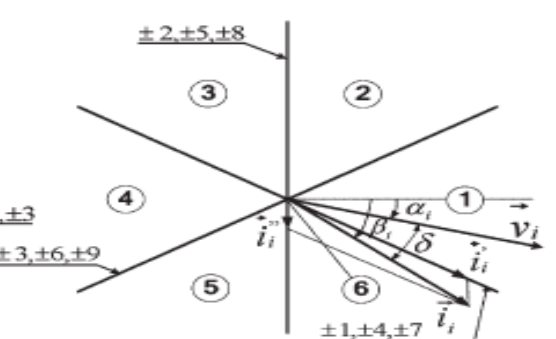

(b)

Fig. 4. (a) Output line-to-neutral voltage vector. (b) Input current and voltage vectors at different sectors.

Similar to Case 1 , to generate the desired voltage vector $\underset{v}{ }$ ' and to maintain the desired different phase angle $\delta$ between the input current vector and the input voltage vector, the suitable SCs selected are $-7,+8,+1,-2$, and zero SCs with the following duty ratios: 
$d_{1}=2 q \sin [\alpha o-(k v-1) \Pi / 3] \sin \left[\frac{\Pi}{6}+(\alpha i-\delta-(k i-2) \Pi / 3)\right] / \sqrt{3} \cos (\delta)$

$d_{2}=2 q \sin [\alpha o-(k v-1) \Pi / 3] \sin \left[\frac{\Pi}{6}-(\alpha i-\delta-(k i-2) \Pi / 3)\right] / \sqrt{3} \cos (\delta)$

$d_{3}=2 q \sin \left[\frac{k v \Pi}{3}-\alpha o\right] \sin \left[\frac{\Pi}{6}+(\alpha i-\delta-(k i-2) \Pi / 3)\right] / \sqrt{3} \cos (\delta)$

$d_{4}=2 q \sin \left[\left(\frac{k v \Pi}{3}-\alpha o\right] \sin \left[\frac{\Pi}{6}-(\alpha i-\delta-(k i-2) \Pi / 3)\right] / \sqrt{3} \cos (\delta)\right.$

$\mathrm{d}_{5}=1-2 \mathrm{q} \cos [\alpha o-\{(2 k v-1) \Pi / 6\}] \cos [\alpha i-\delta-(k i-2) \Pi / 3] / \sqrt{3} \cos (\delta)$

Where $d_{1}, d_{2}, d_{3}, d_{4}$ and $d_{5}$ are the duty ratios of four active SCs (in this case, $-7,+8,+1$, and -2 ) and zero SC, respectively.

Table III shows all possible switching patterns if the desired input current vector and input voltage vector are located in different sectors.

TABLE III

SWITCHING PATTERNS FOR THE INPUT VOLTAGE AND CURRENT VECTORS LOCATED IN THE DIFFERENT SECTORS

\begin{tabular}{|c|c|c|c|c|c|c|c|}
\hline & \multicolumn{6}{|c|}{ Output voltage vetor sector } \\
\hline & & 1 & 2 & 3 & 4 & 5 & 6 \\
\hline \multirow{6}{*}{ 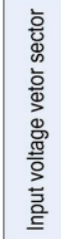 } & 1 & $-7+8+1-2$ & $+9-7-3+1$ & $-8+9+2-3$ & $+7-8-1+2$ & $-9+7+3-1$ & $+8-9-2+3$ \\
\hline & 2 & $+4-5-7+8$ & $-6+4+9-7$ & $+5-6-8+9$ & $-4+5+7-8$ & $+6-4-9+7$ & $-5+6+8-9$ \\
\hline & 3 & $-1+2+4-5$ & $+3-1-6+4$ & $-2+3+5-6$ & $+1-2-4+5$ & $-3+1+6-4$ & $+2-3-5+6$ \\
\hline & 4 & $+7-8-1+2$ & $-9+7+3-1$ & $+8-9-2+3$ & $-7+8+1-2$ & $+9-7-3+1$ & $-8+9+2-3$ \\
\hline & 5 & $-4+5+7-8$ & $+6-4-9+7$ & $-5+6+8-9$ & $+4-5-7+8$ & $-6+4+9-7$ & $+5-6-8+9$ \\
\hline & 6 & $+1-2-4+5$ & $-3+1+6-4$ & $+2-3-5+6$ & $-1+2+4-5$ & $+3-1-6+4$ & $-2+3+5-6$ \\
\hline \multicolumn{2}{|c|}{ Duty ratios } & $d_{1} d_{2} d_{3} d_{4}$ & $d_{1} d_{2} d_{3} d_{4}$ & $d_{1} d_{2} d_{3} d_{4}$ & $d_{1} d_{2} d_{3} d_{4}$ & $d_{1} d_{2} d_{3} d_{4}$ & $d_{1} d_{2} d_{3} d_{4}$ \\
\hline
\end{tabular}

3.3 Maximum Compensated Angle

The duty ratio of the zero SC has to be positive to validate the DSVM method. Considering the fact that $d_{5} \geq 0$, we obtain (20) from (14) and (19)

$q \leq \sqrt{3} \cos (\delta) / 2$

From (20), the maximum voltage transfer ratio is inferred to be $\sqrt{3} \cos (\delta) / 2$, and the well-known maximum voltage transfer ratio becomes $\sqrt{ } 3 / 2$ at $\delta=0$.

As seen from (20), corresponding to each voltage transfer ratio $q$, there exists a possible maximum compensated displacement angle between the desired input current vector and the input voltage vector. However, the new DSVM method is only validated if the input voltage vector leads the input current vector to one sector, i.e., $\delta \leq$ $\pi / 3$. The maximum compensated angle is given by

$\boldsymbol{\delta} \max =\left[\cos ^{-1}\left(\frac{2 q}{3}\right), \quad \sqrt{3} / 4 \leq \mathrm{q} \leq \sqrt{3} / 2\right.$

$[\Pi / 3, \quad 0<\mathrm{q} \leq \sqrt{3 / 4}$.

Fig. 5 shows the relationship between the maximum compensated angle and the voltage transfer ratio representing the reference output voltage of the load demands.

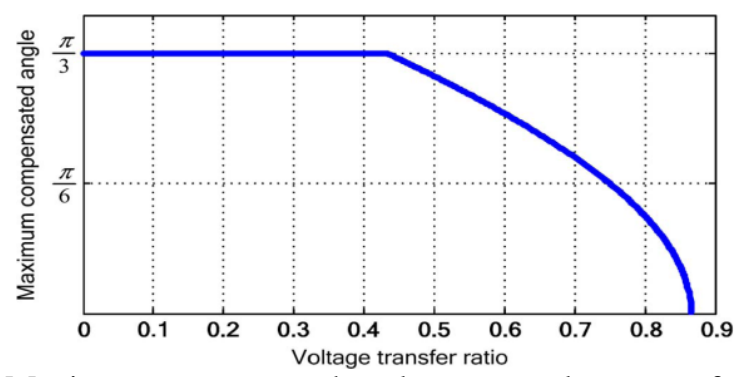

Fig.5. Maximum compensated angle versus voltage transfer ratio.

\section{IPF Compensation Algorithms Using the New DSVM Method}

4.1 Compensation Algorithm Based on the Optimal Compensated Angle

From the filter characteristics explained in Section II, the main input current variation directly impacts the displacement angle between the main input phase voltage and the main input current. 
To overcome this effect, a power factor compensation algorithm based on the optimal compensated angle (compensation algorithm I) is proposed. The optimal compensated angle is calculated in (8); its value depends on the main input current and main input voltage magnitudes, fundamental frequency, and $L C$ values. Fig. 6 shows the proposed online block diagram. The compensated displacement angle is periodically updated at each compensating period Tpf (Tpf >> Ts), where Ts is the PWM sampling period.

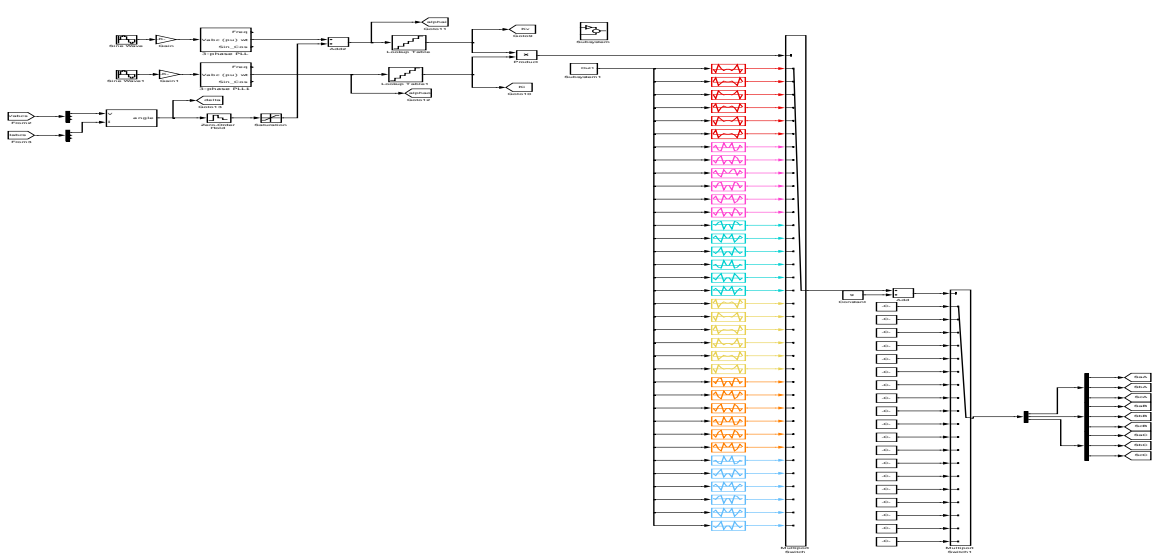

Fig.6. Simulation diagram of compensation algorithm I based on the optimal compensated angle.

The advantages of compensation algorithm I are as follows.

1) It improves the main power factor produced by the new DSVM method without changing the output control.

2) It reduces the main input current magnitude due to a smaller reactive power supply.

In compensation algorithm I, the estimation of the compensated angle is dependent on many parameters that impact on the accuracy of the optimal compensated angle estimation.

\subsection{Compensation Algorithm Based on a PI Controller}

To overcome the drawbacks in compensation algorithm I, another IPF compensation algorithm using a PI controller is proposed: compensation algorithm II. In compensation algorithm II, a sine value of the displacement angle $\psi \mathrm{i}$ between the input line-to-neutral voltage vector and the corresponding input line current vector is chosen to control the IPF in Fig. 7.

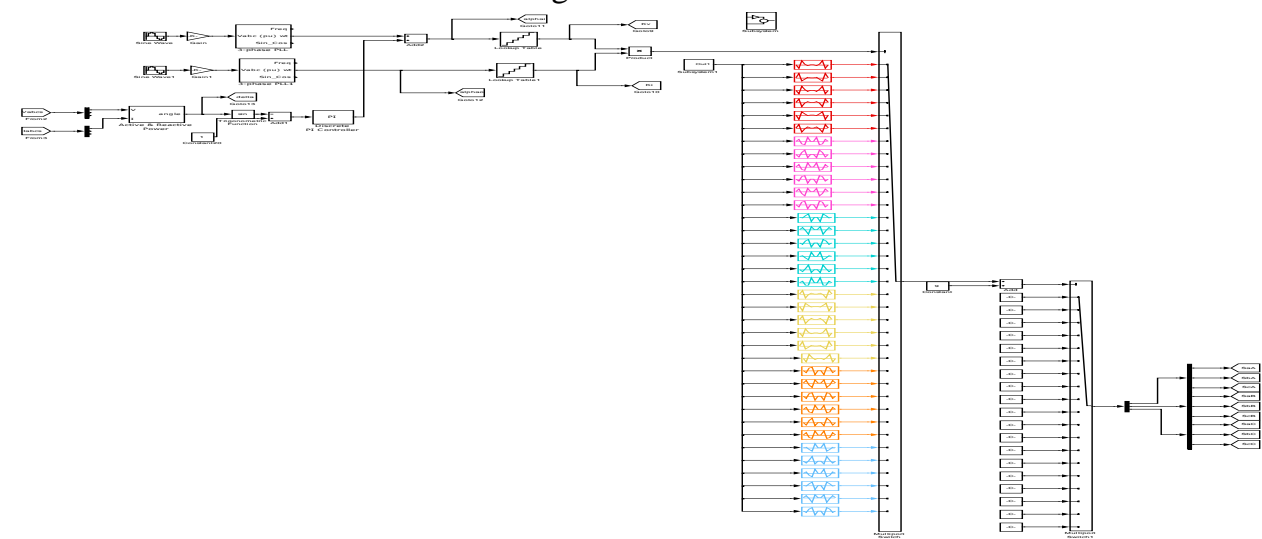

Fig.7. Simulation diagram of compensation algorithm II based on using a PI controller.

The unity power factor at the power supply side of the MC is intrinsically satisfied if the value of $\sin (\psi \mathrm{i})$ is maintained close to zero.

The proposed PI controller is noted by

Where $\Delta \mathrm{e}=\sin (\psi \mathrm{i}) \mathrm{ref}-\sin (\psi \mathrm{i})$ est.

$$
\boldsymbol{\delta} c o m p=(\mathrm{K} p+\mathrm{K} i s) \Delta e
$$

The limiter of the PI controller is always updated with the new maximum compensated angle $\delta$ max

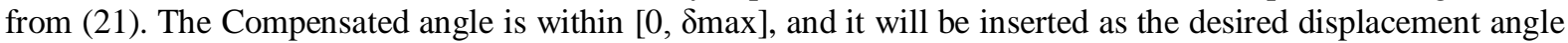
between the input current and the voltage in the new DSVM method. In the case of the IPF compensation, the stable response is more important than the fast response. Thus, PI gains selected for the proposed algorithm are 
recommended: The kp gain is low, and the ki gain is not so large to avoid large overshoot and to have stable performance.

With this proposed algorithm, the compensating period required to maintain a high power factor is as the same as the PWM sampling period $(\mathrm{Tpf}=\mathrm{Ts})$. As the output changes, the PI still functions well in terms of the steady-state and dynamic performance achievement of the input side. Furthermore, this proposed algorithm is independent on the input filter and power supply parameters, which are quite sensitive during practical operations.

\section{Simulation Results and Discussion}

Simulation was carried out on a three-phase $R L$ load using PSIM 6.0 software. The simulation parameters for the $R L$ load were as follows:

1) Power supply (line-to-neutral voltage): $100 \mathrm{~V} / 60 \mathrm{~Hz}$;

2) Three-phase $R L$ load: $26 \Omega, 12 \mathrm{mH}$.

The input filter was designed with $\mathrm{L}=1.4 \mathrm{mH}, \mathrm{C}=22.5 \mu \mathrm{F}$, and $\mathrm{Y}$ connection. The PWM frequency was $10 \mathrm{kHz}$, and simulation results were obtained under a constant $\mathrm{V} / \mathrm{f}$ condition.

Fig. 8 shows the simulation results of the conventional DSVM method at reference output voltage $q=$ $0.7, f o=70 \mathrm{~Hz}$. The input current of the $\mathrm{MC}$ is in phase with the input line-to-neutral voltage. However, the main power supply current lags behind the input line-to-neutral voltage due to the input low-pass filter. The IPF obtained here is only 0.912 .
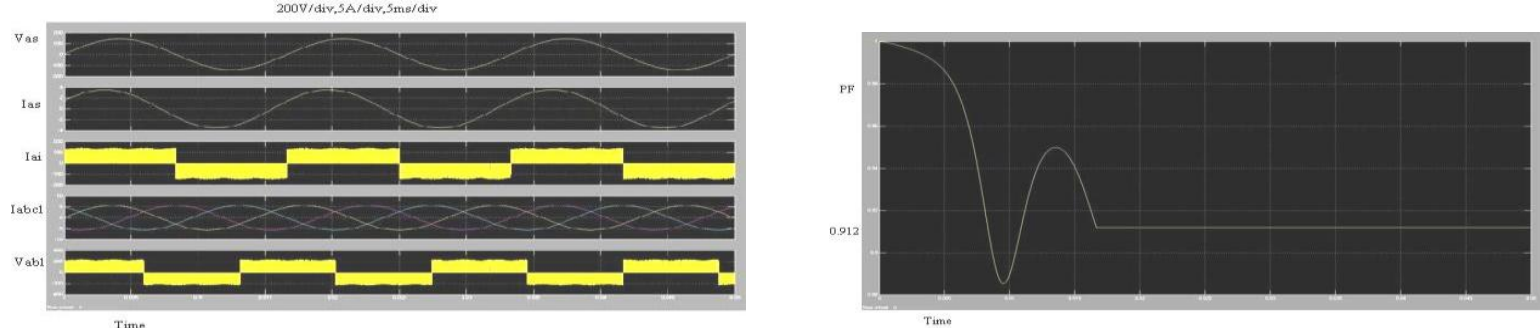

Fig. 8. Input/output waveforms of the MC at reference output voltage $q=0.7, f o=70 \mathrm{~Hz}$ with the conventional DSVM method $(p f=0.912)$.

In order to determine the differences between the conventional DSVM method and our new DSVM method, the same reference output voltage was applied using compensation algorithm II shown in Fig. 9. Due to the optimal compensated angle achieved by the PI controller, the main input current at the power supply is in phase with the line-to-neutral input voltage, while the MC satisfies the reference output voltage presented in the figure by the output currents and line-to-line output voltage.
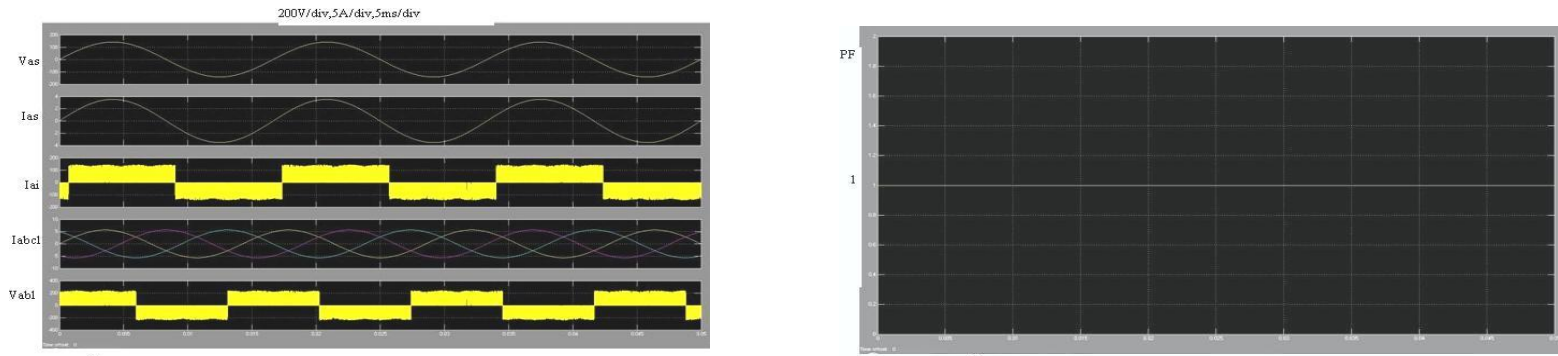

Fig. 9. Input/output waveforms of the MC at reference output voltage $q=0.7, f o=70 \mathrm{~Hz}$ with compensation algorithm II $(p f=1$. $)$

Fig.10(a)-(c) shows the steady-state performances at reference output voltage $\mathrm{q}=0.4$, fo $=40 \mathrm{~Hz}$ of the conventional DSVM method, compensation algorithm I, and compensation algorithm II, respectively. The input currents and voltage waveforms in Fig. 10(a) present the IPF at 0.589. The input currents and voltage waveforms in Fig. 10(b) and (c) describe the MC operations with the optimal compensated angle for compensation algorithms I and II, respectively. The power factors for both algorithms are achieved at unity.

As easily seen in the two cases $(q=0.7, f o=70 \mathrm{~Hz}$ and $q=0.4$, fo $=40 \mathrm{~Hz})$, the optimal compensated angles are smaller than the maximum allowable compensated angles shown in Fig. 5. Therefore, the desired unity power factor can be obtained. 


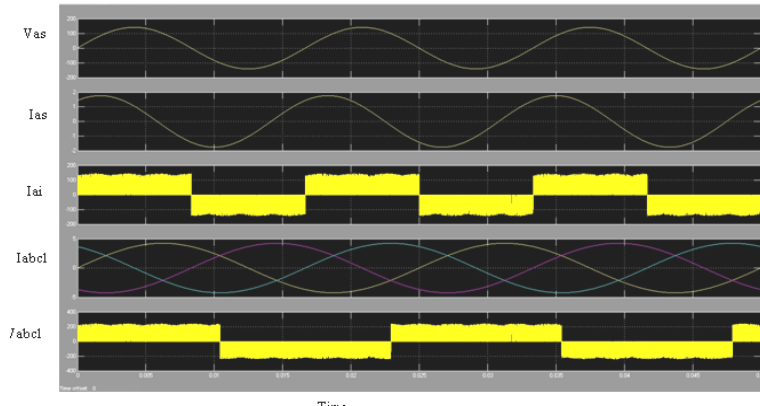

Time

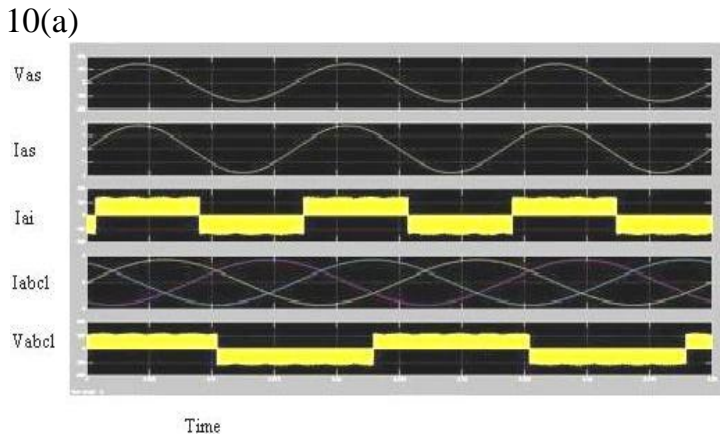

10(b)

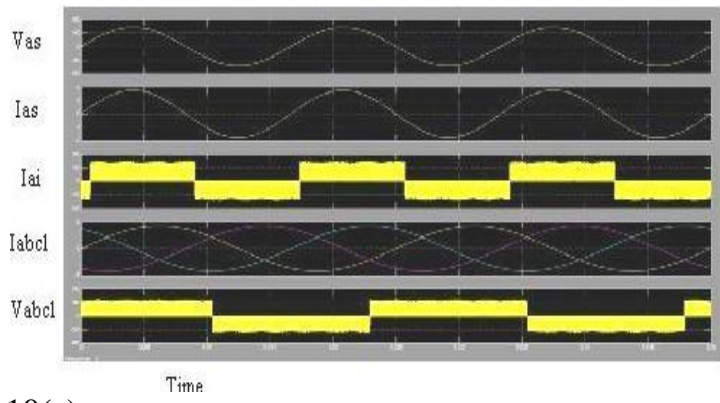

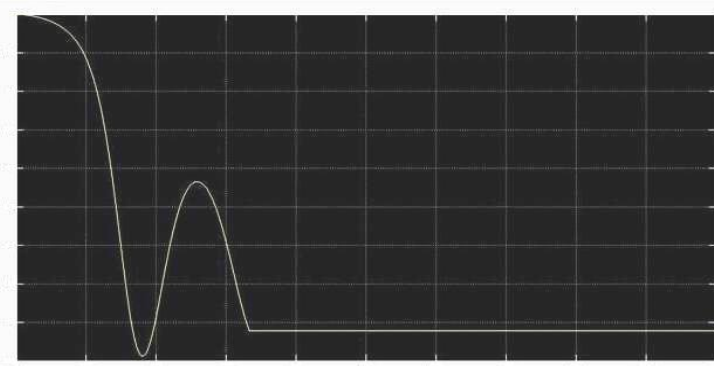

Time

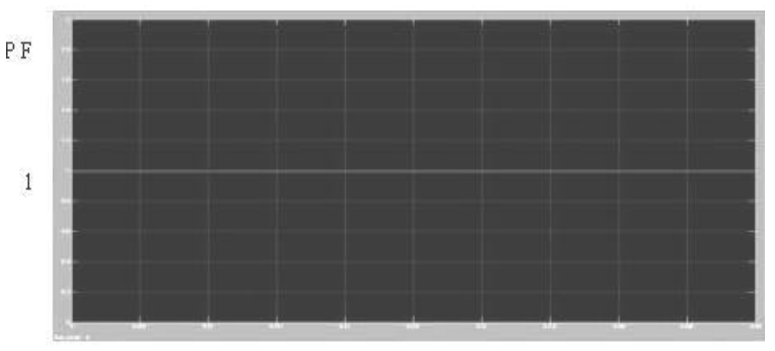

Time

PF

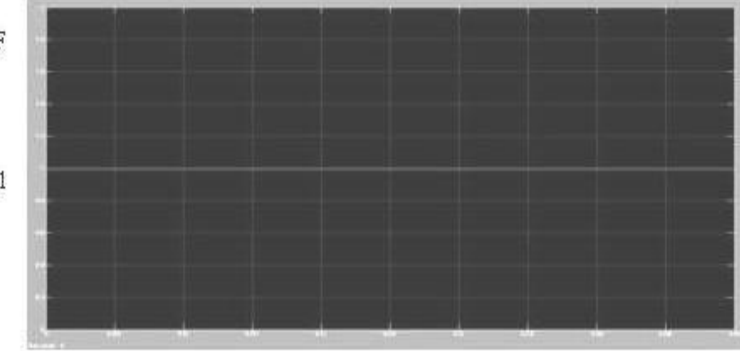

Time

Fig.10. Input waveforms of the MC at reference output voltage $\mathrm{q}=0.4$, fo $=40 \mathrm{~Hz}$. (a) Conventional DSVM method ( $\mathrm{pf}=0.589)$. (b) Compensation algorithm I $(\mathrm{pf}=1.0)$. (c) Compensation algorithm II ( $\mathrm{pf}=1.0)$.

The simulation results of reference output voltage $\mathrm{q}=0.25$, fo $=25 \mathrm{~Hz}$ are shown in Fig. 11. As plotted in Fig. 5, the required optimal compensated angle is higher than the maximum compensated angle $\pi / 3$. Consequently, the maximum compensated angle is applied to compensate the IPF, and the highest IPF (0.445) can be obtained, while only 0.27 is obtained with no compensation in the conventional DSVM method.

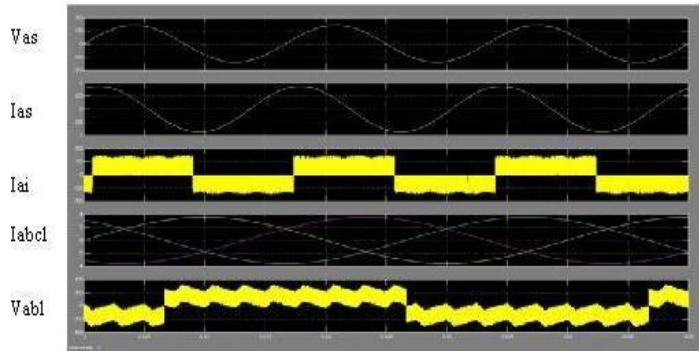

Time

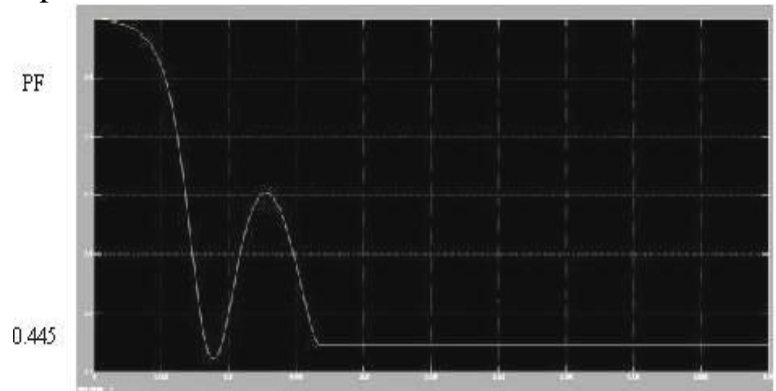

Time

Fig.11. Input/output waveforms of the MC at reference output voltage $q=0.25$, fo $=25 \mathrm{~Hz}$ with the new DSVM method $(\mathrm{pf}=0.445)$.

Observing our new DSVM method's results, we can see that the magnitude of the input current decreases significantly due to the minimum reactive power supply utilization resulting from the proposed IPF compensation algorithms.

Comparison of the IPFs obtained from the conventional DSVM method and the compensation algorithms' using our new DSVM method is shown in Fig. 12. With a constant V/f for the RL load, the higher output load corresponds to the higher voltage transfer ratio (q). The compensated angles at steady state from 
both compensation algorithms are shown in Fig. 12(a). With the high output voltage region $q \geq 0.35$, the IPFs obtained from compensation algorithms I and II are significantly increased to almost unity compared to the lower values obtained from the conventional DSVM method. The difference of the optimal compensated angles obtained from the two compensation algorithms is very small, approximately $0.02 \mathrm{rad}$ shown in Fig. 12(b).
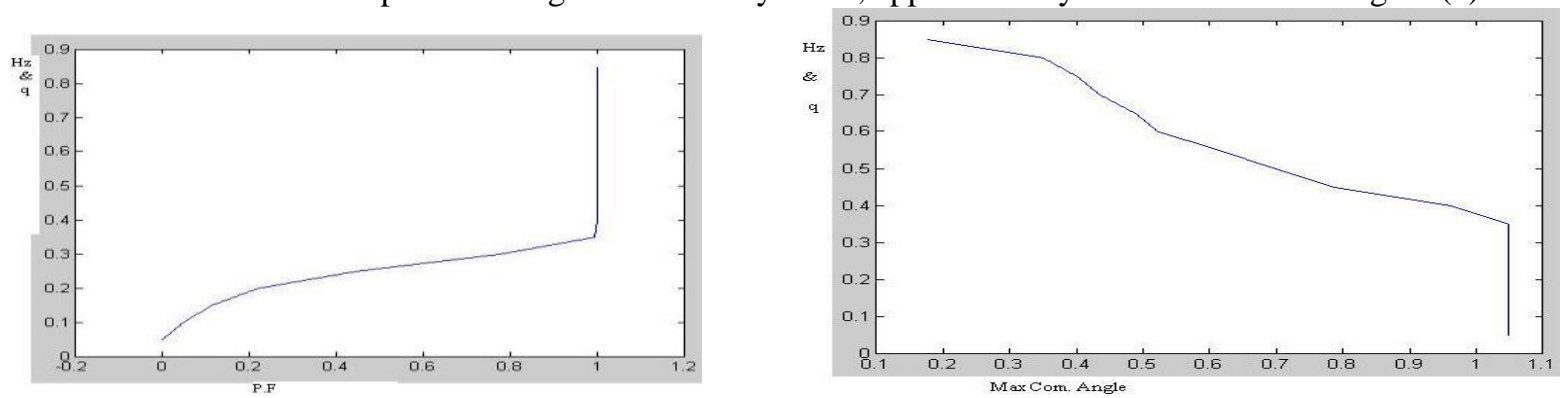

Fig.12. IPFs and compensated angles under constant $V / f$. (a) IPF. (b) Compensated angle.

For the middle output voltage region $0.2 \leq \mathrm{q}<0.35$, the IPF is much higher compared to the one obtained from the conventional DSVM, due to using the maximum compensated angle $\pi / 3$, to both compensation algorithms. At very low output voltage region $\mathrm{q}<0.2$, the output load currents are getting smaller, and the input currents (ii) of the MC synthesized from the output load currents are much smaller than the filter current (ifc) shown in Fig. 1. Moreover, the filter current is the dominant component of the main input current (is). In this case, it is not expected for the compensated angle to significantly improve the main IPF regardless of the maximum compensated angle obtained from compensation algorithm II. As explained in Section 2, compensation algorithm I is based on the approach of unity power factor assumption, and compensation algorithm I can only give the higher power factor due to a certain compensated angle calculated from (8), shown in Fig. 12(b). For more illustrations, simulation result in Fig. 13 shows the waveforms of the filter and MC at reference output voltage $\mathrm{q}=0.15$ with the maximum compensated angle in compensation algorithm II. Because the fundamental waveform of the MC's input current (ii_fund) is so small compared to the fundamental waveform of the filter capacitor's current (ifc_fund), the main input current (is $\approx$ ifc_fund + ii_fund) cannot have a major effect to improve the IPF.

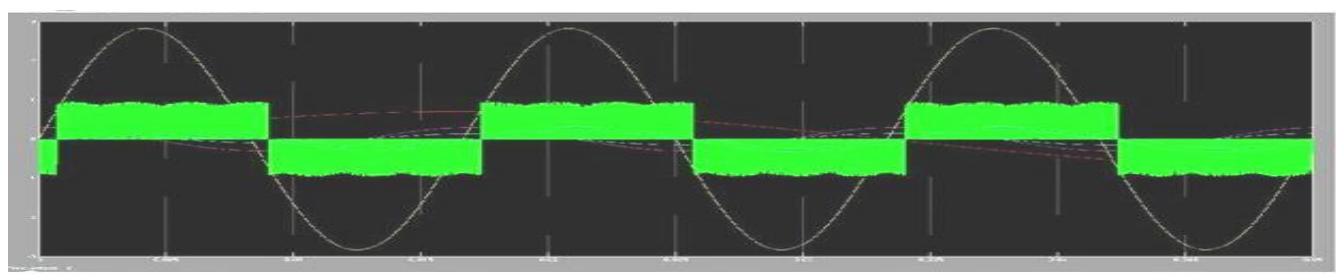

Fig.13.Waveforms of the filter and $\mathrm{MC}$ at reference output voltage $\mathrm{q}=0.15$, fo $=15 \mathrm{~Hz}$ with the maximum compensated angle in compensation algorithm II.

Fig. 14 shows the input/output waveforms of the MC using a PI controller under the new DSVM method during the load change from reference output voltage $\mathrm{q}=0.4$, fo $=40 \mathrm{~Hz}$ to $\mathrm{q}=0.7$, fo $=70 \mathrm{~Hz}$. The main input current is controlled in phase with the line-to-neutral input voltage after a short transient period, and the IPF is still kept at unity after the load change condition. This operation clearly illustrates the effective performance of the proposed algorithm under dynamic load conditions.

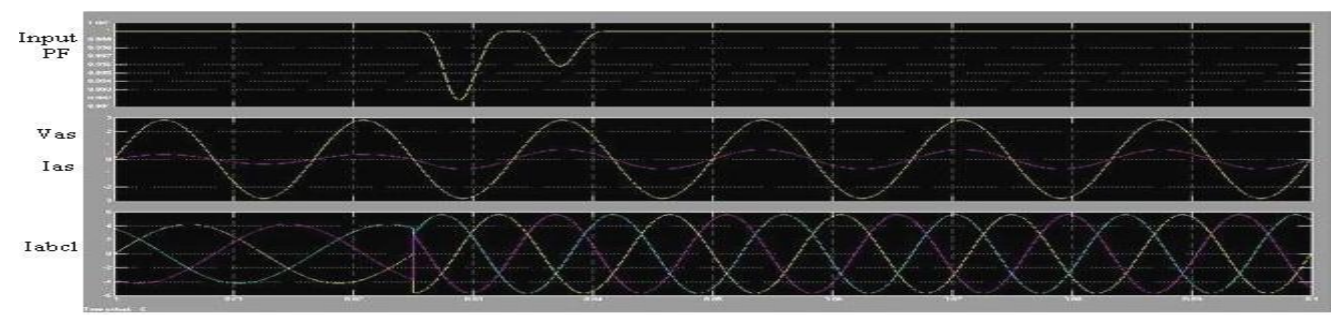

Fig.14.IPF and input/output waveforms of the $\mathrm{MC}$ as the load changes from $\mathrm{q}=0.4$, fo $=40 \mathrm{~Hz}$ to $\mathrm{q}=0.7$, fo $=$ $70 \mathrm{~Hz}$.

Compensation algorithm I using the compensated angle calculated from (8) and compensation algorithm II using a PI controller were both simulated with the reference output voltage $q=0.7, f o=70 \mathrm{~Hz}$. The compensation algorithms were applied at $20 \mathrm{~ms}$ for two cases, and the simulation results are shown in Fig. 15(a) and (b), respectively. 
Matrix Converter Using Direct Svm Method With Input Power Factor Compensation
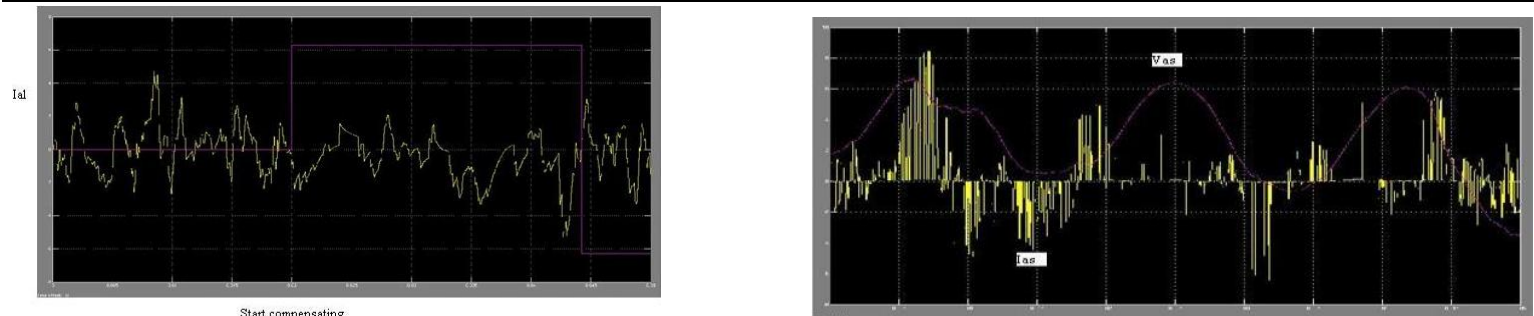

$15(a)$

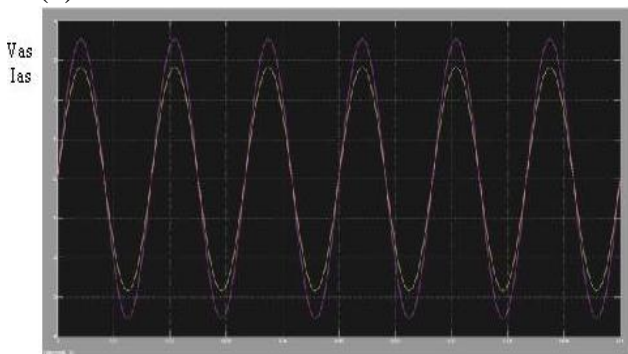

Time

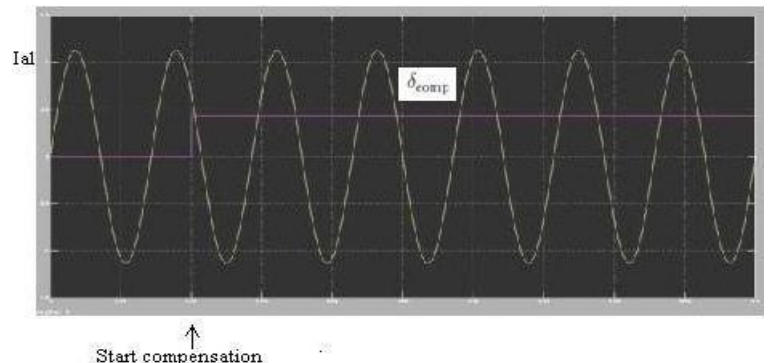

15(b)

Fig. 15. Input/output waveforms of the MC during the compensation process at reference output voltage $q=0.7$, fo $=70 \mathrm{~Hz}$. (a) Compensation algorithm I. (b) Compensation algorithm II.

\section{Conclusion}

This paper proposes a new DSVM technique for matrix converter in terms of improving input power factor at the main power supply side to be unity. Previously, the effect of input filter to the main power factor is just considered at the rated output power of filter/MC/Load system, the near unity power factor at the lower output power is not correct anymore, especially the light load. With this new DSVM method, the near unity main input power factor region is enlarged effectively, beside the higher power factor at the lower output power region, the smaller input current magnitude was obtained by utilizing the minimum reactive power supply as compared to The Conventional DSVM method. The design of sliding mode vector controllers for ac-ac matrix converters has been presented. The obtained results show a good steady-state and dynamic response, allowing the input, as well as the output power factor regulation. Comparison of performances between the two algorithms was carried out and explained in details. The steady-state and transient responses of the proposed algorithms were tested in simulation.

\section{References}

[1] E.Watanabe, S. Ishii, E. Yamamoto, H. Hara, J.-K. Kang, and A.M. Hava, "High performance motor drive using matrix converter," in Proc. IEE Seminar Adv. Induction Motor Control (Ref. No. 2000/072), May 2000, pp. 711-716.

[2] C. Klumpner, P. Nielsen, I. Boldea, and F. Blaabjerg, "A new matrix converter motor (MCM) for industry applications," IEEE Trans. Ind. Electron., vol. 49, no. 2, pp. 325-335, Apr. 2002.

[3] T. F. Podlesak, D. C. Katsis, P. W. Wheeler, J. C. Clare, L. Empringham, and M. Bland, "A 150-kVA vector-controlled matrix converter induction motor drive," IEEE Trans. Ind. Appl., vol. 41, no. 3, pp. 841-847, May/Jun. 2005.

[4] P. W. Wheeler, J. Rodriguez, J. C. Clare, L. Empringham, and A.Weinstein, "Matrix converters: A technology review," IEEE Trans. Ind.Electron., vol. 49, no. 2, pp. 276-288, Apr. 2002.

[5] P. W. Wheeler, H. Zhang, and D. A. Grant, "A theoretical, and practical consideration of optimised input filter design for a low loss matrix converter," in Proc. 9th Int. Conf. Electromagn. Compat., Sep. 1994, pp. 138-142.

[6] P. Wheeler and D. Grant, "Optimized input filter design, and low-loss switching techniques for a practical matrix converter," Proc. Inst. Elect.Eng.-Elect. Power Appl., vol. 144, no. 1, pp. 53-60, Jan. 1997. [7] D. Casadei, G. Serra, A. Tani, and L. Zarri, "Stability analysis of electrical drives fed by matrix converters," in Proc. IEEE ISIE, Jul. 2002, vol. 4, pp. 1108-1113.

[8] D. Casadei, G. Serra, A. Tani, and L. Zarri, "Effects of input voltage measurement on stability of matrix converter drive system," Proc. Inst. Elect. Eng.-Elect. Power Appl., vol. 151, no. 4, pp. 487-497, Jul. 2004.

[9] D. Casadei, G. Serra, A. Tani, A. Trentin, and L. Zarri, "Theoretical, and experimental investigation on the stability of matrix converters," IEEE Trans. Ind. Electron., vol. 52, no. 5, pp. 1409-1419, Oct. 2005.

[10] D. Casadei, J. Clare, L. Empringham, G. Serra, A. Tani, P. W. Wheeler, and L. Zarri, "Large-signal model for the stability analysis of matrix onverters," IEEE Trans. Ind. Electron., vol. 54, no. 2, pp. 939-950, Apr. 2007.

[11] J. Wang and M. Bouazdia, "Influence of filter parameters/topologies on stability of matrix converter-fed permanent magnet brushless motor drive systems," in Proc. IEEE Int. Elect. Machines and Drives Conf.,May 2009, pp. 964-970.

[12] S. F. Pinto and J. F. Silva, "Input filter design for sliding mode controlled matrix converters," in Proc. 32nd IEEE Annu. Power Electron. Spec. Conf., Jun. 2001, vol. 2, pp. 648-653.

[13] S. F. Pinto and J. F. Silva, "Direct control method for matrix converters with input power factor regulation," in Proc. 35th IEEE Power Electron. Spec. Conf., Jun. 2004, vol. 3, pp. 2366-2372.

[14] K. Yamada, T. Higuchi, E. Yamamoto, H. Hara, T. Sawa, M. M. Swamy, and T. Kume, "Filtering techniques for matrix converters to achieve environmentally harmonics drives," in Proc. 11th EPE, Dresden, Germany, 2005.

[15] H.-H. Lee, H. M. Nguyen, and T.-W. Chun, "New direct-SVM method for matrix converter with main input power factor compensation," in Proc. 34th IEEE IECON, Nov. 10-13, 2008, pp. 1281-1286.

[16] N. Burany, "Safe control of four quadrant switches," in Conf. Rec. IEEE IAS Annu. Meeting, 1989, pp. 1190-1194 